\title{
A commentary on "Patient-defined goals for the treatment of fecal incontinence: a qualitative analysis among women attending a urogynecology clinic"
}

\author{
Dominique Malacarne Pape ${ }^{1}$ \\ Received: 17 September 2020 / Accepted: 26 October 2020 / Published online: 9 January 2021 \\ (C) The International Urogynecological Association 2021
}

This was a qualitative analysis conducted at a large academic tertiary referral center. The main objective of this study was to gain information regarding patient goals for fecal incontinence management among women presenting for care. The aim of this trial was to better understand treatment goals, as defined by the patients. Through this understanding, the main aim was to develop a conceptual framework capturing the range of desired therapeutic endpoints in order to direct more comprehensive treatment for fecal incontinence.

One hundred patients with 230 unique goals met the inclusion criteria. Patients were characterized as having fecal incontinence alone, fecal incontinence plus prolapse symptoms, fecal incontinence and urinary complaints or reported all three disorders. Three patient-reported goals were documented prior to visit. Goals were de-identified, and content analysis was performed by two independent researchers to identify common themes; discrepancies were resolved by a third reviewer. Themes were coded and organized until a consensus was reached for categories and sub-categories. Demographically, this was a largely white, non-Hispanic population with $79 \%$ having diagnoses of prolapse, urinary complaints or another pelvic floor disorder in addition to fecal incontinence at the time of initial visit. The five domains identified as key concepts of treatment goals included Emotional Status, Functional Status, Concurrent PFDs, Care Seeking and Treatment Aspirations. Within the Emotional Status domain, three subdomains of Fear/Worry/Anxiety, Embarrassment and Self-Image emerged. The reduction of fear related to

Dominique Malacarne Pape

dominique.malacarnepape@wmchealth.org

1 Division of Advanced Urogynecology and Female Pelvic Medicine and Reconstructive Surgery, New York Medical College, New York, NY, USA one's fecal incontinence remained a constant theme. In terms of Functional Status, the three main subdomains included Activities of Daily Living, Social Engagement and Sexual Function. Goals were focused around increasing baseline/ normal activity and improving general intimacy issues. Overactive Bladder, Urinary Retention, Urinary Tract Infection, Prolapse and Pelvic Pain were the five subdomains of Concurrent PFDs. Goals around Urinary Tract Infection and Pelvic Pain were most emphasized. Within the CareSeeking domain, women expressed desire for education and treatment information most often. The conceptual framework constructed from this analysis captures the intimate relationship between pelvic floor disorders and emotion.

In summary, the researchers identified various common patient-reported goals among female patients with fecal incontinence as well as concomitant pelvic floor disorders. This in turn allowed for the development of a conceptual model illustrating how pelvic floor disorder presence drives care seeking and overall state of emotional and functional status of a patient. Furthermore, this framework depicts how all of these factors contribute to treatment objectives. This results in a more holistic view of fecal incontinence and therapeutic options that patients seek in order to better facilitate comprehensive care of the fecal incontinence patient.

Publisher's note Springer Nature remains neutral with regard to jurisdictional claims in published maps and institutional affiliations. 